\title{
Una Aplicación con Información Geográfica, de Apoyo a la Comercialización de Productos Orgánicos de una Cooperativa de Toacazo-Ecuador
}

\section{A Web Platform with Geographic Information, to Support Trade of Organic Products in a Farmers Cooperative from Toacazo-Ecuador}

\author{
Alex Cevallos Culqui \\ Universidad Técnica de Cotopaxi, Departamento de Ingeniería Informática y \\ Sistemas Computacionales, Latacunga, Ecuador. Av. Simón Rodríguez s/n Sector \\ San Felipe. alex.cevallos@utc.edu.ec
}

Sergio F. Ochoa

Universidad de Chile, Departamento de Ciencias de la Computación, Santiago, Chile. Beauchef 851, Edificio Norte, Sector Santiago Centro. sochoa@dcc.uchile.cl

\section{Gustavo Rodríguez Bárcenas}

Universidad Técnica de Cotopaxi, Departamento de Ingeniería Informática y Sistemas Computacionales, Latacunga, Ecuador. Av. Simón Rodríguez s/n Sector San Felipe.gustavo.rodriguez@utc.edu.ec

\section{Rosa Galleguillos Pozo}

Universidad Técnica de Ambato, Departamento de Ingeniería en Sistemas, Electrónica e Industrial, Ambato, Ecuador. Av. Colombia y Chile Sector Ingahurco. rosagalleguillos@gmail.com

\section{RESUMEN}

En los últimos años se ha incrementado el consumo de alimentos orgánicos, salud y ecología los principales motivos. Varios países latinoamericanos por su biodiversidad y producción tradicional, se han ido convirtiendo en proveedores de productos orgánicos, muchos de ellos son pequeños productores, que intentan colocar sus productos en el mercado, desafortunadamente su distancia a los centros urbanos, limita la conexión entre la oferta y la demanda. El presente proyecto desarrolla una plataforma web de información geográfica, que busca fortalecer el canal de comunicación y comercio entre productores y clientes. Las necesidades de estos actores se han determinado en base a la realidad de una cooperativa de pequeños productores de Toacazo, dedicados al comercio de productos orgánicos. La solución involucra 
cuatro ámbitos del proceso de comercialización: (1) Registro, ingreso georeferenciado de clientes actuales y potenciales; (2) Venta, búsqueda-selección de productos; (3) Pago, online de la orden de venta; (4) Integración, de la producción individual de los productores. Todo esto con una metodología de trabajo que se basó en el establecimiento de un proceso iterativo, permitiendo mejorar las funcionalidades de la aplicación paulatinamente. La aplicación ha centralizado información geográfica del cliente y el productor, permitiendo el intercambio de información georeferencial de ambos actores, importante para localizar el lugar de entrega de una orden de venta. Se ha logrado que la cooperativa disponga de un nuevo canal de comercio para sus productos, por medio del cual no sólo clientes actuales, sino también clientes potenciales, pueden hacer su pedido y pagarlo de forma online.

Palabras clave: Información geográfica; georeferencial; productos orgánicos; ecología; productores; comercio electrónico; aplicaciones web; pago online.

\section{ABSTRACT}

In the last years, organic food consumption has increased, health and ecology the main reasons. Several Latin American countries for its biodiversity and traditional production, have become suppliers of organic products. Many of these suppliers are small farmers, who try to set their products on the market, unfortunately their distance from urban centers restricts the connection between offer and demand. In this project we have developed a web platform with geographical information to strengthen the channel of communication and commerce between farmers and customers. The needs of these actors are determined, considering the reality of a cooperative of small farmers from Toacazo (Cotopaxi, Ecuador) engaged in trade of organic products. The solution involves four areas of the trading process: (1) Registration, the recording of georeferenced information about current and potential customers; (2) Sale, search and selection of products; (3) Payment online of the sale order; (4) Integration of individual production of farmers. All this, with a work methodology based on an iterative process, which has allowed to improve each application functionality gradually. The application has centralized geographic information of customers and farmers, allowing the exchange of georeferential information between these two actors; this has been important to locate the delivery place of a sale order. This work has allowed to these farmers to count on a new trade channel for their products. Through this cannel, current and potential customers can place their order and pay online.

Keywords: Geo-referenced information; GIS; organic products; ecology; farmers; e-commerce; web application; online payment. 


\section{INTRODUCCIÓN}

La revolución industrial fue el hito que marcaría celeridad en el ritmo de producción de la sociedad, incentivando el mayor consumo. Esta época trajo consigo técnicas de producción que fortalecieron las economías a escala, así se adoptó el concepto de "más por menos". Según los años pasaban, muchas áreas productivas se industrializaron, y en la época de los cincuenta bajo la metáfora "revolución verde, combatir el hambre" fue el turno del campo, así la producción agrícola se triplica, a través de cultivos convencionales y químicos. Este fenómeno hace que en Ecuador y en el mundo, el mercado agrario sea abastecido por dos tipos de producción: el convencional y el orgánico. La globalización golpea fuertemente a la agricultura familiar-orgánica, debido a la competencia dispareja con la agroindustria. Una producción con alta tecnología es difícilmente igualable para aquellos que usan sólo sus manos para trabajar (Cevallos, 2011; Elegido, 1975).

Es así, como hoy en día el método de cultivo para la obtención de un producto agrícola empieza a tener importancia, comercialmente los productos orgánicos tienden a valorizarse y diferenciarse de los convencionales. Es decir, la producción orgánica empieza a tener importancia, pero lamentablemente, este tipo de producción enfrenta ciertas limitaciones como: la falta de organización de los productores, el restringido uso de tecnología por parte de estas personas y los limitados canales de comercio a los que ellos tienen acceso. Motivos como éstos provocan que los agricultores orgánicos se encuentren en una situación relativamente débil para comercializar sus productos, obteniendo por lo tanto términos contractuales desventajosos y precios bajos (Damiani \& Silveri, 2003; Del Bosque et al., 2012).

Para tratar de sortear esta limitante, el objetivo de esta investigación es diseñar e implementar una aplicación web, que embeba un sistema de información geográfica, la cual busca fortalecer el canal de comunicación y comercio entre productores y clientes. Permitiendo que pequeños agricultores orgánicos, tengan una mejor visibilidad de su ubicación y producción agrícola. La solución involucra automatizar cuatro ámbitos del proceso de comercialización: (1) Registro, ingreso de información georeferenciada de clientes actuales y potenciales; (2) Integración, de la producción individual de los productores; (3) Venta, búsqueda-selección de productos; (4) Pago, online de la orden de venta.

Como una forma de delimitar el alcance de la aplicación, la solución está enfocada a las particularidades de la cooperativa Probio. Esta cooperativa se encuentra ubicada en la parroquia Toacazo, provincia de Cotopaxi, a una hora de la capital ecuatoriana Quito. La cooperativa posee líderes con estudios superiores que impulsan este tipo de agricultura. Ellos están abiertos al uso de nuevas tecnologías. Un ejemplo de su apertura es su actual recepción de pedidos vía mail. Los productores asociados a través de esta cooperativa, aprovechando la fortaleza del incremento del consumo de productos orgánicos y avizorando la amenaza de los intermediarios, se han integrado para dedicarse al comercio de hortalizas y frutas orgánicas. A futuro se buscará la forma de extender el sistema para dar apoyo a cooperativas con otras realidades.

En las siguientes secciones, se describe el método adoptado para el cumplimiento de la meta, así como el proceso de desarrollo de la aplicación. 
Finalmente en la última sección se reportan los resultados y la discusión de los mismos en relación a las principales implicaciones con respecto al uso de la información de la plataforma web para la comercialización de productos orgánicos de la cooperativa de productores orgánicos Probio.

\section{MÉTODOS}

Para la presente investigación, considerando la realidad de la cooperativa de pequeños productores de Toacazo, dedicados al comercio de productos orgánicos, se desarrolla una plataforma web que acarrea un conjunto de acciones que se muestran a continuación:

1. Registro tradicional y geo-referencial de clientes potenciales y actuales.

2. Búsqueda, selección de productos.

3. Pago online de la orden.

4. Integración de la producción individual de los productores.

Para desarrollar esta plataforma y representarla en un caso de estudio se han considerado los siguientes aspectos (Izaurralde, 2013; Merchán et al., 2008; Rivadeneira, 2012):

- La determinación de aspectos funcionales para el desarrollo del sistema, a través de historias de usuario y la toma de requerimientos.

- Consideración de la arquitectura, diseño e ingeniería en las funcionalidades de cada módulo de la implementación del sistema.

- Pruebas de funcionalidad y aceptación de cada uno de los módulos que conforman el sistema.

Todo esto con una metodología de trabajo que se basó en el establecimiento de un proceso iterativo, permitiendo mejorar las funcionalidades de la aplicación paulatinamente.

\section{Contexto de Implementación}

El Sistema web de información Geográfica de apoyo a la comercialización de Productos Orgánicos (SGPO), ha sido implementado en una estructura tres capas MVC (Modelo Vista Controlador), para lo cual se empleó el Framework Spring, ésto con el fin de segmentar la interfaz de la lógica de negocio y brindar mayor seguridad al entorno (ver Figura 1-A). Todas las herramientas de construcción han sido OpenSource, las mismas que permitieron el manejo de aplicaciones geográficas y la implementación de la lógica de negocio de la plataforma, para el almacenamiento de datos geográficos, se utilizó el motor de base de datos PostgreSQL ${ }^{1}$ con su extensión PostGis².

\footnotetext{
${ }^{1}$ Sistema Gestor de Base de Datos

${ }^{2}$ Extensión de PostgreSQL para almacenamiento de datos geográficos
} 


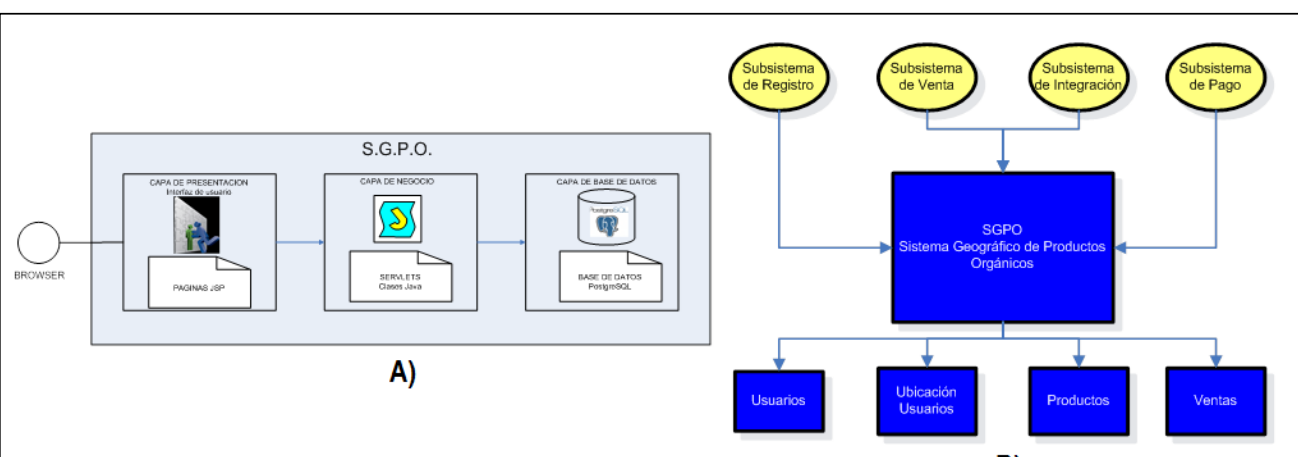

B)

Figura 1 Estructura y Subsistemas de SGPO. La Figura 1-A muestra las capas de trabajo de la arquitectura del Framework Spring. La Figura 1-B describe las funcionalidades de la aplicación.

Considerando las necesidades de la cooperativa de productores orgánicos en estudio, el SGPO es estructurado con cuatro subsistemas de negocio fundamentales que se describen en la Figura 1-B.

1) Subsistema de Registro: Administra el registro de datos de cuenta y ubicación geográfica del cliente actual o potencial. Se obtienen datos de usuarios y ubicación de usuarios.

2) Subsistema de Venta: Búsqueda geográfica de productos por la categoría: productor, fruta u hortaliza. El usuario administra la selección de productos en una canasta de compras. Se obtiene una orden de compra en estado inicial, la cual finalizará cuando la orden sea pagada.

3) Subsistema de Pago: Una vez confirmada la canasta de compras, se ejecuta a su pago online por medio de PayPal. Registrados los datos de pago y confirmada la transacción, se procede al control de stock de productos y el cambio de la orden de venta a su estado final.

4) Subsistema de Integración: Unifica la producción individual de cada uno de los productores pertenecientes a la cooperativa. Con el fin de satisfacer pedidos que superen la capacidad individual de los productores.

\section{Desarrollo}

Los cuatro subsistemas establecidos en la aplicación se los desarrolló considerando la secuencia de usabilidad (1.Registro, 2.Venta, 3.Pago, 4. Integración) que el usuario tiene con la aplicación. Las razones para considerar la secuencia de los subsistemas fueron dos: primero, mitigar los riesgos de mayor envergadura en la parte inicial y segundo, obtener una funcionalidad paulatina que pueda seguir siendo probada.

Considerando la globalidad del desarrollo de la aplicación y la metodología de trabajo definida inicialmente, se plantearon dos instancias en el proceso de 
construcción (ver Tabla 1). En la primera, la implementación de cada subsistema en forma individual e incremental y en la segunda, un refinamiento de todos los subsistemas en forma conjunta e incremental. Cada incremento y cada instancia fueron añadiendo valor a las funcionalidades de la aplicación.

Tabla 1 Instancias de Implementación

\begin{tabular}{|c|c|}
\hline Primera Instancia & Segunda Instancia \\
\hline $\begin{array}{l}\text { Propósito: } \\
\text { - Desarrollar cada uno de los } \\
\text { subsistemas en forma individual e } \\
\text { incremental. } \\
\text { - Obtener un modelo que pueda ser } \\
\text { usado y probado de forma } \\
\text { individual por un administrador de } \\
\text { la cooperativa. }\end{array}$ & $\begin{array}{l}\text { Propósito: } \\
\text { - Receptar como entrada los cuatro } \\
\text { subsistemas, en un estado } \\
\text { considerable de uso, para fortalecer } \\
\text { su integración. } \\
\text { - Encontrar y mejorar falencias en las } \\
\text { funcionalidades de los subsistemas. }\end{array}$ \\
\hline $\begin{array}{l}\text { Iteración 1: } \\
\text { Centrado en la estructura de la } \\
\text { funcionalidad y en los objetivos del } \\
\text { subsistema. }\end{array}$ & $\begin{array}{l}\text { Iteración 1: } \\
\text { Se definió un plan de pruebas para la } \\
\text { validación de los subsistemas en } \\
\text { conjunto. }\end{array}$ \\
\hline $\begin{array}{l}\text { Iteración 2: } \\
\text { Ajusta la organización de las clases } \\
\text { empleadas en paquetes, y segmenta la } \\
\text { estructura inicial del subsistema. }\end{array}$ & $\begin{array}{l}\text { Iteración 2: } \\
\text { Las pruebas de funcionalidad } \\
\text { preparadas, son ejecutadas con la } \\
\text { ayuda de un focus group. }\end{array}$ \\
\hline $\begin{array}{l}\text { Iteración 3: } \\
\text { Mejora el diseño de la interfaz de } \\
\text { presentación, para ofrecer mejor } \\
\text { usabilidad al usuario. }\end{array}$ & $\begin{array}{l}\text { Iteración 3: } \\
\text { Registro de observaciones y realización } \\
\text { de correcciones. }\end{array}$ \\
\hline
\end{tabular}

Definidas las iteraciones, que establecen un camino para cada uno de los subsistemas, se fue construyendo la trayectoria hacia la obtención de un nivel de madurez considerable, acorde a las características del destino deseado para cada uno. A continuación se detalla la trayectoria recorrida con cada uno de los subsistemas.

1) Subsistema de Registro.- El resultado final del subsistema es obtener una sesión que guarde la identificación del usuario que se ha registrado. Para conseguir esta meta fue necesario que el subsistema recorra las iteraciones presentadas en la Tabla 2. Por lo tanto este subsistema ha sido implementado para que el usuario pueda:

- Registrar sus datos de cuenta de usuario y generar una sesión a través de la identificación y validación de sus datos de cuenta (user y password) registrados.

- Registrar sus datos de ubicación geográfica, a través de su participación con la cartografía presentada. 
Tabla 2 Descripción Iteraciones del subsistema de registro

\begin{tabular}{|c|c|c|}
\hline \multicolumn{3}{|c|}{ Subsistema de Registro } \\
\hline Iteración 1 & Iteración 2 & Iteración 3 \\
\hline $\begin{array}{l}\text { Se identifica dos riesgos } \\
\text { a ser mitigados: }\end{array}$ & $\begin{array}{c}\text { Se evalúan alternativas } \\
\text { para mitigar los riesgos } \\
\text { encontrados: }\end{array}$ & $\begin{array}{l}\text { Integración de } \\
\text { tecnologías: }\end{array}$ \\
\hline $\begin{array}{l}\text { 1. Tipo de estructura } \\
\text { tecnológica Web: el } \\
\text { Framework elegido } \\
\text { prevalecería en los }\end{array}$ & $\begin{array}{l}\text { 1. Se efectuaron } \\
\text { pruebas con los } \\
\text { Frameworks Spring y }\end{array}$ & $\begin{array}{l}\text { 1. Selección del } \\
\text { Framework Spring por } \\
\text { sus cualidades de } \\
\text { flexibilidad e integración }\end{array}$ \\
\hline $\begin{array}{l}\text { subsistemas siguientes, } \\
\text { en esta iteración para el } \\
\text { registro tradicional, se } \\
\text { trabaja con el Framework } \\
\text { Struts. }\end{array}$ & $\begin{array}{l}\text { Django. El primero con } \\
\text { un alto nivel de } \\
\text { madurez de flexibilidad } \\
\text { e integración. El } \\
\text { segundo de fácil } \\
\text { conectividad con datos }\end{array}$ & $\begin{array}{l}\text { 2. La tecnología definida } \\
\text { para la estructura } \\
\text { geográfica fue OSM } \\
\text { (OpenStreetMaps). }\end{array}$ \\
\hline $\begin{array}{c}\text { 2. Tipo de tecnología } \\
\text { geográfica: inicialmente } \\
\text { se buscaba trabajar con } \\
\text { cartografía }\end{array}$ & $\begin{array}{l}\text { geográficos pero con } \\
\text { limitantes en } \\
\text { integración. }\end{array}$ & $\begin{array}{l}\text { Para el enlace de OSM } \\
\text { con el Framework } \\
\text { Spring, se ha hecho uso } \\
\text { de las librerías }\end{array}$ \\
\hline $\begin{array}{c}\text { independiente al } \\
\text { disponer de shapes } \\
\text { geográficos de la zona, } \\
\text { esto con PostGIS y } \\
\text { Geoserver. }\end{array}$ & $\begin{array}{l}\text { 2. Se efectúan pruebas } \\
\text { de las librerías de } \\
\text { cartografía de } \\
\text { OpenStreetMaps } \\
\text { (OMS) y GoogleMaps }\end{array}$ & $\begin{array}{l}\text { OpenLayers. Así es } \\
\text { como los contextos de } \\
\text { registro tradicional y } \\
\text { geográfico son } \\
\text { fusionados. }\end{array}$ \\
\hline $\begin{array}{c}\text { Los dos contextos } \\
\text { distaban mucho de ser } \\
\text { fusionados. La rigidez } \\
\text { que brindaban ambos } \\
\text { entornos no generaba un } \\
\text { buen ambiente de } \\
\text { trabajo }\end{array}$ & $\begin{array}{l}\text { Se identifica un } \\
\text { Framework con } \\
\text { flexibilidad de } \\
\text { integración y librerías } \\
\text { OpenSource para } \\
\text { manejar cartografía. }\end{array}$ & \\
\hline
\end{tabular}

2) Subsistema de Venta.- Este subsistema recibe como entrada una sesión que contiene datos de identificación del usuario. El subsistema ha sido implementado para que el usuario pueda efectuar las siguientes actividades:

- Buscar información tradicional y geográfica de clientes, productores, frutas y/o hortalizas.

- Administrar la selección de productos en una canasta de compras. La orden de venta puede estar relacionada a uno o varios productores. La administración implica añadir, modificar y retirar la cantidad de productos que se desee de la canasta de compras.

El resultado final de este subsistema fue obtener una orden de compra que contenga el listado y cantidad de productos que el usuario desea. Para 
conseguir estas metas fue necesario que el subsistema recorra las iteraciones presentadas en la Tabla 3.

Tabla 3 Descripción de Iteraciones del subsistema de venta

\begin{tabular}{|c|c|c|}
\hline \multicolumn{3}{|c|}{ Subsistema de Venta } \\
\hline Iteración 1 & Iteración 2 & Iteración 3 \\
\hline $\begin{array}{l}\text { Se identifican dos } \\
\text { necesidades: } \\
\text { 1. Un modelo de datos } \\
\text { que soporte un catálogo }\end{array}$ & $\begin{array}{c}\text { Se preparó la capa de } \\
\text { presentación para que } \\
\text { despliegue los datos } \\
\text { cargados en la base de } \\
\text { datos. }\end{array}$ & $\begin{array}{c}\text { Se afinan los queries } \\
\text { HQL para mejorar el } \\
\text { tiempo de respuesta, } \\
\text { en la entrega de } \\
\text { datos. }\end{array}$ \\
\hline $\begin{array}{l}\text { pueda ser identificado } \\
\text { individualmente por } \\
\text { productor y de forma } \\
\text { conjunta para toda la } \\
\text { Cooperativa. } \\
\text { 2. Un modelo que permita } \\
\text { controlar y administrar } \\
\text { la orden de venta, a } \\
\text { través de una sesión }\end{array}$ & $\begin{array}{c}\text { Se preparó los Queries } \\
\text { HQL (Hibernate Query } \\
\text { Language) que se } \\
\text { usaron en la búsqueda } \\
\text { de información } \\
\text { tradicional y geográfica } \\
\text { de clientes, } \\
\text { productores, frutas y/o } \\
\text { hortalizas. }\end{array}$ & $\begin{array}{l}\text { Se diseña una } \\
\text { canasta de } \\
\text { productos, amigable } \\
\text { y de fácil uso, que } \\
\text { permite añadir, } \\
\text { modificar y/o retirar } \\
\text { productos de una } \\
\text { orden de venta. }\end{array}$ \\
\hline $\begin{array}{c}\text { activa en el sistema. } \\
\text { En base a estas } \\
\text { necesidades se diseñó una } \\
\text { estructura de clases. }\end{array}$ & $\begin{array}{l}\text { Se preparó a la capa } \\
\text { de presentación para } \\
\text { que reciba peticiones } \\
\text { de búsqueda y } \\
\text { despliegue datos } \\
\text { geográficos en } \\
\text { cartografía. }\end{array}$ & $\begin{array}{l}\text { Se prepara la capa } \\
\text { de negocio para } \\
\text { recibir peticiones de } \\
\text { mantenimiento en la } \\
\text { orden de venta. }\end{array}$ \\
\hline
\end{tabular}

3) Subsistema de Pago.- Recibe como entrada una orden de compra que contiene el listado y cantidad de productos que el usuario pretende adquirir. Este subsistema ha sido implementado para que el usuario pueda realizar las siguientes actividades:

- Confirmar su canasta de compras y presentar el detalle de su orden de compra.

- Pagar de forma online su orden de compra, por medio de una cuenta PayPal.

Para conseguir estas metas fue necesario que el subsistema recorra las iteraciones presentadas en la Tabla 4. 
Tabla 4 Descripción de Iteraciones del subsistema de pago

\begin{tabular}{|c|c|c|}
\hline \multicolumn{3}{|c|}{ Subsistema de pago } \\
\hline Iteración 1 & Iteración 2 & Iteración 3 \\
\hline $\begin{array}{c}\text { Se identifica la necesidad } \\
\text { de establecer una conexión } \\
\text { con PayPal, para lo cual se } \\
\text { realiza las siguientes } \\
\text { acciones: } \\
\text { Se habilita una opción para } \\
\text { que el usuario confirme su } \\
\text { canasta de compras, con } \\
\text { esta confirmación se } \\
\text { cambia el estado (creada, } \\
\text { confirmada, pagada) de la } \\
\text { orden de compra para que } \\
\text { proceda con el pago } \\
\text { PayPal. } \\
\text { Se analiza los diferentes } \\
\text { tipos de pago y estructuras } \\
\text { de conexión de PayPal, en } \\
\text { esta iteración se prepara la } \\
\text { capa de negocio para el } \\
\text { levantamiento de conexión } \\
\text { con la estructura Adaptive } \\
\text { Payments. }\end{array}$ & $\begin{array}{l}\text { Se realizan pruebas y } \\
\text { confirma el uso de la } \\
\text { estructura de pago } \\
\text { Express Checkout, con } \\
\text { las siguientes } \\
\text { consideraciones: } \\
\text { Validación de datos } \\
\text { complementarios de la } \\
\text { orden de venta. } \\
\text { Enlace con el API de } \\
\text { PayPal y envío de la } \\
\text { orden de venta, para que } \\
\text { el usuario proceda con el } \\
\text { pago o cancelación de la } \\
\text { compra. } \\
\text { La aplicación recibe a } \\
\text { nivel de lógica de negocio } \\
\text { la confirmación o } \\
\text { revocación de la compra. }\end{array}$ & $\begin{array}{c}\text { Se valida en la } \\
\text { aplicación a nivel de } \\
\text { lógica de negocio para } \\
\text { el cierre de la orden de } \\
\text { venta, con las } \\
\text { siguientes } \\
\text { consideraciones: } \\
\text { Validar que la } \\
\text { respuesta del API de } \\
\text { PayPal corresponda a } \\
\text { una orden cuyo pago } \\
\text { haya finalizado con } \\
\text { éxito. } \\
\text { Cambiar la orden de } \\
\text { venta a su estado final. } \\
\text { Reducir el stock de los } \\
\text { productos adquiridos. }\end{array}$ \\
\hline
\end{tabular}

4) Subsistema de Integración.- Para este subsistema no fue importante ningún tipo de información de entrada. Este subsistema permite al usuario:

- Integrar la producción individual de los productores de la cooperativa. En el caso de que la demanda supere la cantidad de producción individual de los productores.

- Administrar la selección de productos considerando el stock integrado de la producción.

Para conseguir estas metas fue necesario que el subsistema recorra las iteraciones presentadas en la Tabla 5. 
Tabla 5 Descripción Iteraciones del subsistema de integración

\begin{tabular}{|c|c|c|}
\hline \multicolumn{3}{|c|}{ Subsistema de Integración } \\
\hline Iteración 1 & Iteración 2 & Iteración 3 \\
\hline $\begin{array}{l}\text { La funcionalidad de este } \\
\text { modelo no representaba } \\
\text { mayor riesgo para el } \\
\text { desempeño de la } \\
\text { aplicación puesto que es } \\
\text { un servicio } \\
\text { complementario para }\end{array}$ & $\begin{array}{l}\text { En esta iteración se } \\
\text { diseñó un procedimiento, } \\
\text { que filtra la cantidad de } \\
\text { producción que posee } \\
\text { cada productor, para } \\
\text { acumularla. }\end{array}$ & $\begin{array}{l}\text { Se preparó a la capa } \\
\text { de presentación, para } \\
\text { que despliegue el } \\
\text { catálogo de productos } \\
\text { con su cantidad de } \\
\text { producción integrada. }\end{array}$ \\
\hline $\begin{array}{c}\text { SGPO. } \\
\text { Sin embargo, se analizó el } \\
\text { modelo de clases y los } \\
\text { atributos necesarios para } \\
\text { su implementación. }\end{array}$ & $\begin{array}{c}\text { El total de esta } \\
\text { integración obtenida se } \\
\text { almacenada en base de } \\
\text { datos. }\end{array}$ & $\begin{array}{c}\text { Para esta } \\
\text { presentación se utilizó } \\
\text { el mismo diseño del } \\
\text { catálogo utilizado en el } \\
\text { subsistema de venta, } \\
\text { donde el usuario } \\
\text { puede añadir, } \\
\text { modificar y/o retirar la } \\
\text { cantidad de productos } \\
\text { que desee. }\end{array}$ \\
\hline
\end{tabular}

\section{RESULTADOS}

Considerando el método de trabajo planificado y la estructura de desarrollo de la aplicación, se define dos instancias de prueba: la primera involucra a cada subsistema de forma individual, y la segunda considera todos los subsistemas de la aplicación en forma conjunta. Con estas consideraciones se planificó un set de pruebas para cada subsistema con un grupo de usuarios productores y clientes con los cuales se ejecuta la inspección de los subsistemas.

La cooperativa definió un representante encargado del seguimiento y validación de las funcionalidades del sitio web. Por el lado del usuario cliente, se trabajó con clientes actuales de confianza de la cooperativa y clientes potenciales que tienen interés en usar el canal de comercio (ver Tabla 6). Para la ejecución del set de pruebas, se publica la aplicación cohesionada en la URL:http://anakena.dcc.uchile.cl:8080/mo/ 
Tabla 6 Usuarios para Pruebas de Inspección

\begin{tabular}{|c|c|c|c|c|c|}
\hline Usuario & $\begin{array}{c}\text { U1 } \\
\text { Camilo Viera }\end{array}$ & $\begin{array}{c}\text { U2 } \\
\text { Norma } \\
\text { Marcalla }\end{array}$ & $\begin{array}{c}\text { U3 } \\
\text { Rene Vela }\end{array}$ & $\begin{array}{c}\text { U4 } \\
\text { Andrea } \\
\text { Idrovo }\end{array}$ & $\begin{array}{c}\text { U5 } \\
\text { Jenny } \\
\text { Zambrano }\end{array}$ \\
\hline Tipo & $\begin{array}{l}\text { Cliente de la } \\
\text { Cooperativa }\end{array}$ & $\begin{array}{c}\text { Representante } \\
\text { de la } \\
\text { Cooperativa }\end{array}$ & $\begin{array}{c}\text { Directivo de } \\
\text { la } \\
\text { cooperativa }\end{array}$ & $\begin{array}{l}\text { Cliente de la } \\
\text { cooperativa }\end{array}$ & $\begin{array}{l}\text { Cliente de la } \\
\text { cooperativa }\end{array}$ \\
\hline \multirow[t]{2}{*}{$\begin{array}{l}\text { Descrip } \\
\text { ción }\end{array}$} & $\begin{array}{l}\text { Profesión: } \\
\text { Turismo }\end{array}$ & $\begin{array}{l}\text { Profesión: } \\
\text { Agrónoma }\end{array}$ & $\begin{array}{l}\text { Profesión: } \\
\text { Agrónoma }\end{array}$ & $\begin{array}{l}\text { Profesión: } \\
\text { Comerciante }\end{array}$ & $\begin{array}{l}\text { Profesión: } \\
\text { Parvularia }\end{array}$ \\
\hline & $\begin{array}{c}\text { Uso Internet: } \\
\text { nivel } \\
\text { avanzado. }\end{array}$ & $\begin{array}{l}\text { Uso Internet: } \\
\text { nivel medio. }\end{array}$ & $\begin{array}{l}\text { Uso Internet: } \\
\text { nivel medio. }\end{array}$ & $\begin{array}{c}\text { Uso Internet: } \\
\text { nivel } \\
\text { avanzado. }\end{array}$ & $\begin{array}{c}\text { Uso Internet: } \\
\text { nivel } \\
\text { avanzado }\end{array}$ \\
\hline
\end{tabular}

Estas actividades definen el flujo de proceso que se ha llevado a cabo para la ejecución de pruebas de SGPO (Figura 2). El proceso deja un camino abierto para la planificación de nuevas iteraciones de pruebas, que proporcionen nuevos incrementos a las características de SGPO. Cada plan de pruebas generado permite registrar observaciones que aportan a mejorar la aplicación.

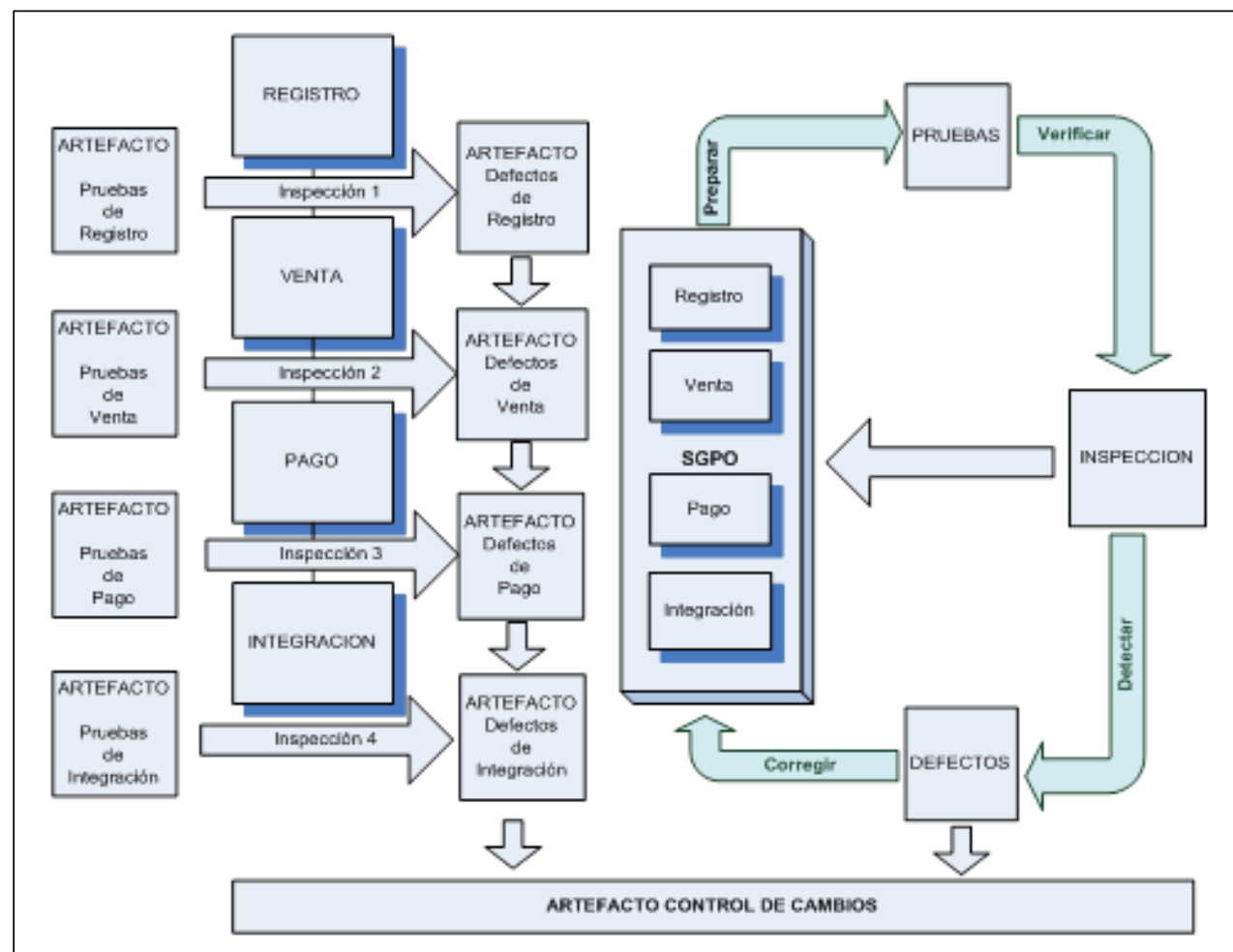

Figura 2 Flujo del proceso de pruebas. Planificación del flujo de trabajo para las pruebas por subsistema y globales a realizarse con la aplicación SGPO. 
Las observaciones y defectos obtenidos en la primera instancia (ver Tabla 7), generaron mayor madurez a la aplicación, también afinaron el nuevo plan de pruebas que consideraba la globalidad de la aplicación. Las pruebas globales se las dirigió al Focus Group descrito en la Tabla 6, los defectos y observaciones que se obtuvieron (Tabla 7), al igual que en la instancia anterior se fueron registrando en el artefacto de control de cambios.

Tabla 7 Observaciones de las pruebas por subsistema y globales

\begin{tabular}{|c|c|c|c|}
\hline \multicolumn{4}{|c|}{ Pruebas por Subsistema } \\
\hline $\begin{array}{c}\text { Subsistema } \\
\text { Registro }\end{array}$ & $\begin{array}{c}\text { Subsistema } \\
\text { Venta }\end{array}$ & $\begin{array}{c}\text { Subsistema } \\
\text { Pago }\end{array}$ & $\begin{array}{l}\text { Subsistema } \\
\text { Integración }\end{array}$ \\
\hline $\begin{array}{l}\text { Alto tiempo de } \\
\text { respuesta al cargar } \\
\text { cartografía. } \\
\text { Inadecuada } \\
\text { usabilidad en el } \\
\text { almacenamiento de } \\
\text { la ubicación } \\
\text { geográfica, el } \\
\text { usuario olvida } \\
\text { guardarla. } \\
\text { Regular } \\
\text { desempeño de la } \\
\text { aplicación con } \\
\text { navegador Firefox. }\end{array}$ & $\begin{array}{c}\text { Incorporar } \\
\text { información del } \\
\text { productor y/o } \\
\text { cliente en los } \\
\text { markers de la } \\
\text { búsqueda de } \\
\text { información } \\
\text { geográfica. } \\
\text { En la } \\
\text { administración de } \\
\text { la canasta de } \\
\text { compras, los } \\
\text { defectos } \\
\text { encontrados } \\
\text { exigían } \\
\text { correcciones de } \\
\text { estética y } \\
\text { validación. }\end{array}$ & $\begin{array}{c}\text { Se valora la } \\
\text { necesidad de } \\
\text { información del } \\
\text { contacto del } \\
\text { cliente. } \\
\text { Antes de hacer el } \\
\text { pago, es } \\
\text { necesario } \\
\text { permitir que el } \\
\text { usuario valide su } \\
\text { ubicación } \\
\text { registrada. }\end{array}$ & $\begin{array}{c}\text { Permitir comparar } \\
\text { entre } \\
\text { cooperativas así } \\
\text { como se puede } \\
\text { hacer } \\
\text { comparación } \\
\text { entre } \\
\text { productores. }\end{array}$ \\
\hline $\begin{array}{l}\text { - Permitir la altern } \\
\text { - } \text { de producto. } \\
\text { - } \quad \text { Replidar datos de } \\
\text { - Volver al las ven } \\
\text { - Vock in }\end{array}$ & $\begin{array}{l}\text { Pruebas } \\
\text { va de búsqueda } \\
\text { icación y contact } \\
\text { registradas de la } \\
\text { al finalizada la se }\end{array}$ & $\begin{array}{l}\text { bales } \\
\text { exto y no por se } \\
\text { ntes de confirma } \\
\text { nana. } \\
\text { a. }\end{array}$ & $\begin{array}{l}\text { ción de categoría } \\
\text { orden de venta. }\end{array}$ \\
\hline
\end{tabular}

Las observaciones y/o defectos se usaron para corregir la aplicación, los cambios ejecutados brindaron mejor usabilidad y calidad a la aplicación. A partir de esta iteración el proceso está abierto a la preparación y refinamiento de nuevos planes de pruebas, para continuar mejorando las funcionalidades de SGPO. 


\section{Funcionalidades de la aplicación}

Desarrollada y validada la aplicación, en la Figura 3 se muestra las secciones de la interfaz principal obtenida, resultado de la cohesión de los cuatro subsistemas planificados y estructurados.

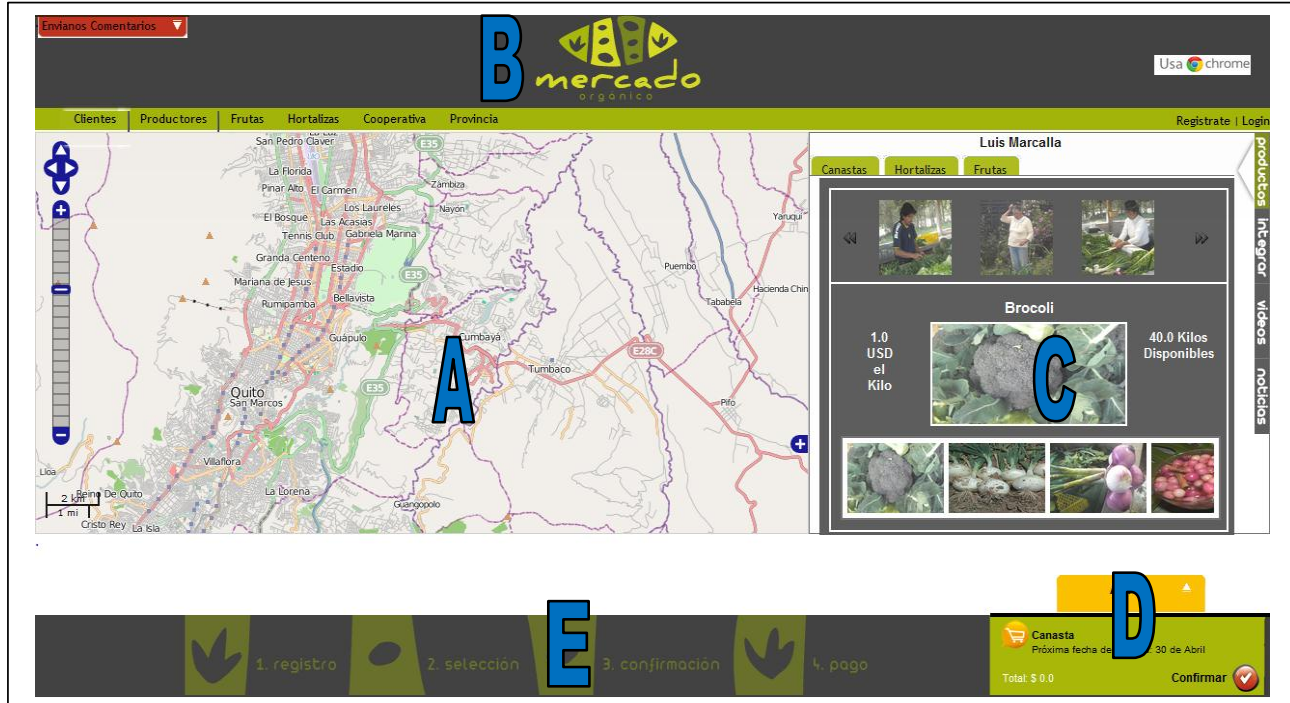

Figura 3 Secciones de la interfaz principal, entre las que se pueden mencionar: A. sección de mapas, B. Sección de cabecera, C. Sección de catálogo, D. Sección de Canasta, E. Sección Estad y F. Sección Flotante.

A. Sección Mapas: Zona donde se despliega la información de cartografía para el usuario, ya sea para determinar la ubicación de un productor o la suya propia.

B. Sección Cabecera: Posee un menú de opciones vinculadas a dos actividades: el registro (derecha) y la búsqueda (izquierda). Cuando no existe una sesión activa el usuario puede registrarse o loguearse si dispone de sus datos de cuenta. También puede buscar información geográfica de acuerdo a las categorías: clientes, productores, frutas, hortalizas, cooperativa, provincia.

C. Sección Catálogo: Zona donde se despliega el listado de productos disponibles de la cooperativa, clasificándolos por productor. Es aquí donde el usuario puede seleccionar los productos deseados para preparar su canasta de compras.

D. Sección Canasta: Lugar donde se van acumulando los productos seleccionados. Aquí el usuario puede modificar el contenido de su canasta. Esta sección también posee el link de confirmación de la canasta. Que se ejecuta cuando el usuario haya decido el contenido de su compra.

E. Sección Estado: Aquí se presenta el estado de la compra con respecto a un usuario. Cuatro son los estados por los cuales el usuario transita hasta finalizar su compra: registro, selección, confirmación y pago. 
F. Sección Flotante: Son ventanas float que presentan información complementaria para cada uno de los subsistemas. Generalmente serán formularios que obtienen información de los usuarios y la entregan al sistema.

1) Subsistema de Registro.- Permite la recepción de datos de productores y clientes, información que se encuentra a la disponibilidad de la cooperativa. Las funcionalidades habilitadas en este subsistema son:

- Administración de datos tradicionales de usuarios: Consiente la creación y modificación de cuentas de usuario que poseen información de productores y clientes (actuales y potenciales).

- Administración de datos geográficos de usuarios: Permite la fijación y edición (en cartografía) de la posición geográfica del usuario; información que aporta a la obtención de su domicilio.

- Identificación de cuenta: El usuario accede al sistema usando el nombre y password con el que se registró, se valida los datos de cuenta y se genera una sesión de trabajo en la aplicación.

La mayor parte de las actividades de este módulo se las efectúa en las secciones mapas y flotante. Para el registro geográfico el usuario utiliza cartografía (ver Figura 4-A) y para el registro tradicional una ventana emergente para registrar sus datos (ver Figura 4-B).

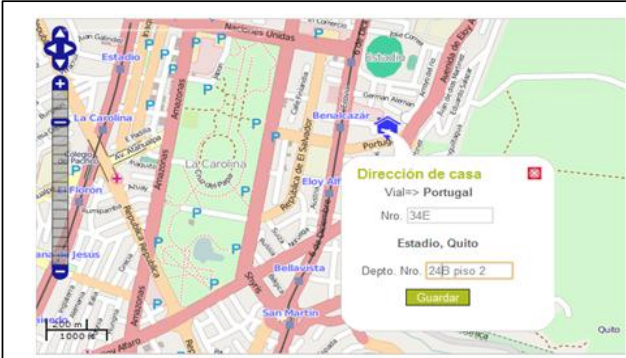

A)

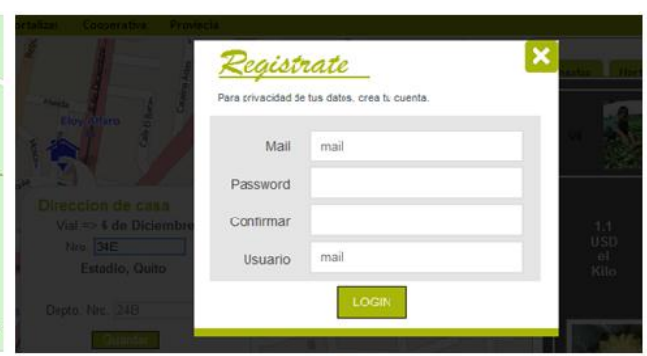

B)

Figura 4 Interfaz de registro geográfico y tradicional. En la Figura 4-A se muestra el registro geográfico del domicilio del usuario, En la Figura 4-B se observa el registro tradicional, una ventana emergente para registrar datos del usuario.

2) Subsistema de Venta.- Permite que usuarios ejecuten búsquedas, dirigidas a encontrar información sobre la producción que posee la cooperativa. También establece un canal de comunicación entre el cliente y la cooperativa, por el cual se entrega información de una canasta de compras deseada. Las funcionalidades habilitadas son:

- Búsqueda de información de la cooperativa: Permite la búsqueda de información en las siguientes categorías: clientes, productores, frutas y hortalizas. Haciendo uso de OSM (OpenStreetMaps), a los resultados de la búsqueda se adiciona información geográfica. 
- Administración de canasta de compras: Se permite la inserción, modificación y eliminación de productos que se ligan a una orden de compra de un usuario, ésta puede estar relacionada a uno o más productores.

La mayor parte de las actividades de este módulo se las efectúa en las secciones catálogo y canasta (Figura 5A). Inicialmente por defecto el sistema despliega en la sección catálogo todos los productos que posee la cooperativa, clasificándolos por productor.

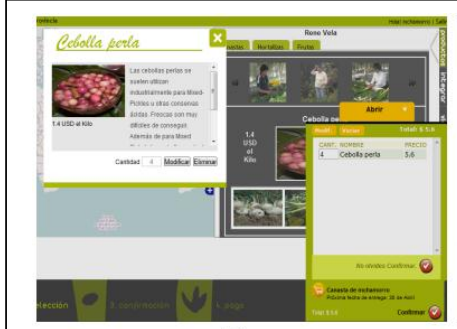

A)

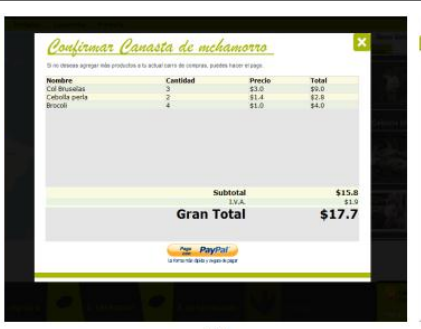

B)

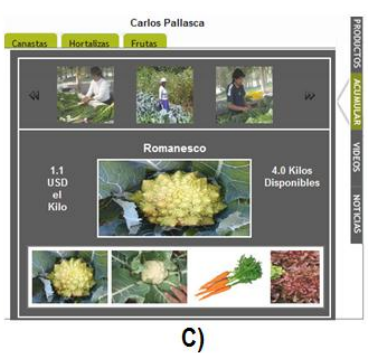

C)

Figura 5 Interfaces de los subsistemas de venta, pago e integración, la Figura 5-A. muestra la gestión del catálogo y canasta de productos, la Figura 5-B presenta la forma de hacer el pago online de la orden de venta que preparó el usuario, la Figura 5-C exhibe la integración de la producción de todos los productores.

3) Subsistema de Pago.- Permite que usuarios que han preparado una canasta de compras, puedan hacer el pago online de su orden (ver Figura 5B). Las funcionalidades habilitadas son las siguientes:

- Confirmación de orden de compra: El usuario puede revisar y confirmar su canasta de compras.

- Pago Online con PayPal: Verificada y confirmada la orden de compra, el usuario puede hacer el pago de forma online con una cuenta PayPal.

4) Subsistema de Integración.- Permite acumular la cantidad de producción individual de los productores pertenecientes a la cooperativa. El usuario puede administrar su canasta de compras, en base a cantidades de stock acumuladas. Las funcionalidades habilitadas son:

- Calcular la producción total de la cooperativa: Se puede totalizar la cantidad de producción que pueden acumular los productores de la cooperativa.

- Administración de canasta de compras: Con cantidades integradas que superan el stock individual de un productor. Se permite la inserción, modificación y eliminación de productos vinculados a una orden de compra de un usuario.

El sistema por medio de la opción integrar, ubicada en la parte vertical derecha de la sección catálogo (ver Figura 5C), permite la integración de la producción de todos los productores. 


\section{COMENTARIOS Y CONCLUSIONES}

Se ha generado una aplicación web que recopila información tradicional y geográfica de clientes y producción orgánica de los productores de la Cooperativa ecuatoriana Probio, permitiendo el comercio electrónico de los productos de la cooperativa. Esto se realizó por medio de una aplicación geográfica (SGPO). El conocimiento de la ubicación del origen de los productos brinda mayor confiabilidad a los clientes para su adquisición. Así como conocer la ubicación de clientes actuales y potenciales facilita la logística de entrega del producto solicitado.

Coincidiendo con resultados de investigaciones similares tales como (Del Bosque et al., 2012; Garrocho, 1998; Girard, 2015) este estudio brindará a la cooperativa el fortalecimiento de sus canales de comercio. Ya que al momento sus ventas están dirigidas únicamente a clientes actuales, con la aplicación, se brinda mayor apertura para la recepción de nuevos clientes potenciales, esto con el fin de incrementar sus ventas. Considerando a la aplicación como una herramienta de promoción y marketing de sus productos.

Otra fortaleza es el hecho de tener una forma de pago online. Para la recepción de los pagos online confirmados en PayPal, se define usar datos de una cuenta designada por la cooperativa, la cual es la responsable de la administración de los ingresos.

Para el cliente, la importancia que tiene la aplicación, está en el tiempo que un usuario emplea para hacer sus compras en la tienda virtual, como también aseveran (Girard, 2015; Rocha et al., 2010; Rosero et al., 2010; Tejada et al., 2011). Al momento el promedio es de quince minutos. En este tiempo el usuario puede registrarse, seleccionar y pagar productos del catálogo de nuestra aplicación, considerando que tenga una cuenta PayPal activa. Este tiempo es aceptable considerando el tiempo y esfuerzo que implica hacer la misma compra en una feria orgánica o supermercado.

Se acuerda con la cooperativa algunas definiciones de negocio importantes, por ejemplo la entrega de las compras registradas en SGPO no será inmediata, se las hará una vez por semana. El stock de cada producto corresponde a un valor constante semanal que el productor lo obtiene con su planificación de cultivo. Después de la entrega semanal, el stock de los productos en la aplicación adopta su estado inicial. 


\section{REFERENCIAS}

1. Cevallos, A. (2011). Un Sistema Web de Información Geográfica, de Apoyo a la Comercialización de Productos Orgánicos de una Cooperativa de Pequeños Productores de Ecuador. Tesis de Magíster en Tecnologías de la Información, Universidad de Chile, Departamento de Ciencia de la Computación, Santiago, Chile.

2. Damiani, O., \& Silveri, P. (2003). La adopción de la agricultura orgánica por parte de los pequeños agricultores de América Latina y el Caribe. Fondo Internacional de Desarrollo Agrícola.

3. Del Bosque, I., Fernández, C., Martín-Forero, L., \& Peréz, E. (2012). Los sistemas de información geográfica y la investigación en ciencias humanas y sociales: Confederación Española de Centros de Estudios Locales. Madrid: Confederación Española de Centros de Estudios Locales.

4. Elegido, M. (1975). "El impacto de la agricultura sobre el medio ambiente". Revista de Estudios Agrosociales, 31-58.

5. Garrocho, C. (1998). "Los sistemas de información geográfica en la geografía médica". Economía Sociedad y Territorio.

6. Girard, M. (2015). "Economía social y comercialización de productos orgánicos en Cusco, Perú.i Pasos iniciales en el desarrollo de una estrategia alternativa?". Eutopía Revista de Desarrollo Económico Territorial.

7. Izaurralde, M. (2013). Caracterización de Especificación de Requerimientos en entornos Ágiles: Historias de Usuario. Córdoba.

8. Merchán, L., Urrea, A., \& Rebollar, R. (2008). "Definición de una metodología ágil de ingeniería de requerimientos para empresas emergentes de desarrollo de software del sur-occidente colombiano". Revista Guillermo de Ockham, 6.

9. Rivadeneira, G. (2012). Metodologías ágiles enfocadas al modelado de requerimientos. Universidad Nacional de la Patagonia Austral, Río Turbio.

10. Rocha, W., Delgado, M., \& Sendra, J. (2010). "Desarrollo de modelos de crecimiento urbano óptimo para la Comunidad de Madrid aplicando métodos de evaluación multicriterio y Sistemas de Información Geográfica". Revista Internacional de Ciencia y Tecnología de la Información Geográfica.

11. Rosero, C., Vasquez, P., \& Cordero, V. (2010). Análisis situacional de la soberanía alimentaria en el contexto de la adaptación al cambio climático en el Ecuador. PNUD.

12. Tejada, C., Edison, J., Jurado Rodriguez, E., Mendoza, I., \& Ortiz Morán, L. (2011). Proyecto de inversión sobre la comercialización de productos orgánicos en la ciudad de Guayaquil. Escuela Superior Politécnica del Litoral, Facultad de Economía y Negocios, Guayaquil. 